\title{
, Structural Coloring of Glass Using Dewetted Nanoparticles and Ultrathin Films of Metals
}

\author{
${ }_{3}$ Renwen Yu, ${ }^{\dagger}$ Prantik Mazumder, ${ }^{\dagger}$ Nick F. Borrelli, ${ }^{\ddagger}$ Albert Carrilero, ${ }^{\dagger}$ Dhriti S. Ghosh ${ }^{\dagger}$ \\ ${ }_{4}$ Rinu A. Maniyara, ${ }^{\dagger}$ David Baker, ${ }^{\ddagger}$ F. Javier García de Abajo, ${ }^{*},{ }^{\dagger}$, and Valerio Pruneri ${ }^{*}, \dagger, \S$ \\ $5{ }^{\dagger}$ ICFO-Institut de Ciencies Fotoniques, The Barcelona Institute of Science and Technology, 08860 Castelldefels (Barcelona), Spain \\ $6{ }^{\ddagger}$ Corning Inc., Sullivan Park, Corning, New York 14830, United States \\ $7{ }^{\S}$ ICREA-Institució Catalana de Recerca i Estudis Avançats, Passeig Lluís Companys 23, 08010 Barcelona, Spain
}

8 S Supporting Information

9 ABSTRACT: Metal nanoparticles have been used for coloring glass since antiquity.

10 Colors are produced by light scattering and absorption associated with plasmon

11 resonances of the particles. Recently, dewetting at high temperature has been

12 demonstrated as a straightforward high-yield/low-cost technique for nanopatterning

13 thin metal films into planar arrays of spherical nanocaps. Here, we show that by simply

14 tuning the contact angle of the metal dewetted nanocaps one can achieve narrow

15 resonances and large tunability compared with traditional approaches such as changing

16 particle size. A vast range of colors is obtained, covering the whole visible spectrum and

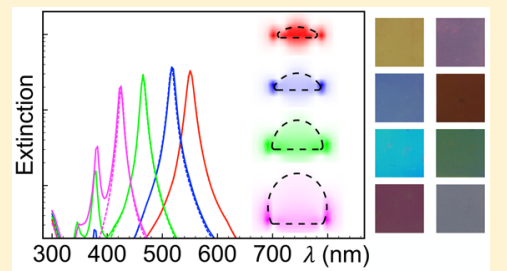

readily controlled by the choice of film thickness and materials. The small size of the particles results in a mild dependence on incidence illumination angle, whereas their high anisotropy gives rise to strong dichroism. We also show color tuning through interference by simply adding an ultrathin metal film at a designated distance from the dewetted particle array. Our measurements are quantitatively well explained through analytical theory, which enables fast optimization of fabrication parameters. Good agreement between theory and experiment requires incorporating the effect of plasmon peak broadening caused by the finite size distribution of the particle. The proposed designs and techniques hold great potential for large-scale production of colored and dichroic glass with application to optical windows, filters, and displays.

24 KEYWORDS: structural colors, dewetting, plasmonics, glass coloring

25 arly evidence of dichroic colored glass can be found as 26 soon as fourth century Rome: the celebrated Lycurgus ${ }_{27}$ Cup, ${ }^{1}$ whose color changes between red and green depending 28 on the illumination angle. We had to wait until recent times to 29 understand that coloring was produced by dispersed silver 30 nanoparticles, whose localized surface plasmon resonances 31 (LSPRs) affect very differently the light spectra in either 32 reflection or transmission. Based on this effect and on the 33 dependence of LSPRs on particle size, shape, and composition, 34 various strategies have been explored for structural color35 ing, $^{2-12}$ including LSPR hybridization in metal nanodisks and 36 nanoholes. $^{13}$

37 Modern dichroic colored glass is however based on thin-film 38 interference, which is widely used in a variety of commercial 39 optical filters ${ }^{14}$ to produce narrow spectral bands for 40 applications such as fluorescence microscopy ${ }^{15}$ and LCD 41 projectors. ${ }^{16}$ On the exploratory side, intense research efforts 42 are being devoted to creating bright structural colors through 43 thin-film interference involving ultrathin metal films

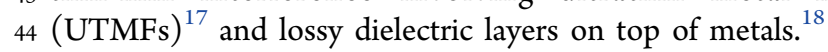

45 In many applications it is crucial to create colors in a 46 transparent substrate (e.g., glass) via surface nanostructuring. ${ }^{19}$ 47 This is a flexible strategy that can be applied to virtually any 48 glass and does not require bulk modifications, such as the 49 addition of inclusions. However, in order to attract industrial interest, it must be low cost and mass scalable. Intensive optical 50 and electron-beam nanolithography are therefore not an 51 option. Instead, dewetting of UTMFs has recently been 52 shown to provide an efficient way of forming metal nano- 53 particles on large silica surfaces. $^{20-22}$ The process consists in 54 depositing a continuous UTMF and heating it to several 55 hundred degrees Celcius, so that the film contiguity disappears 56 and the metal groups together into nanoparticles with 57 characteristic size distributions. Surface energies of UTMF 58 and substrate as well as the interfacial energy between them 59 drive the particle shape into nanocaps of well-determined 60 contact angle $\beta$ with the substrate. ${ }^{23}$ Interestingly, the particle 61 size and surface density can be controlled by the original film 62 thickness, as well as by the duration and temperature of the 63 heating treatment. ${ }^{24}$ Metal dewetting is thus a viable option for 64 simple, low-cost lithography-free surface nanostructuring, 65 which is ideal for industrially scalable applications.

66

Here, we demonstrate the use of dewetted metal nanocaps 67 on transparent silica surfaces to produce and customize 68 structural colors. In particular, we demonstrate for the first 69 time that by changing the contact angle of the nanocaps we can 70 obtain larger tuning and narrower LSPRs than traditional 71

Received: February 10, 2016 

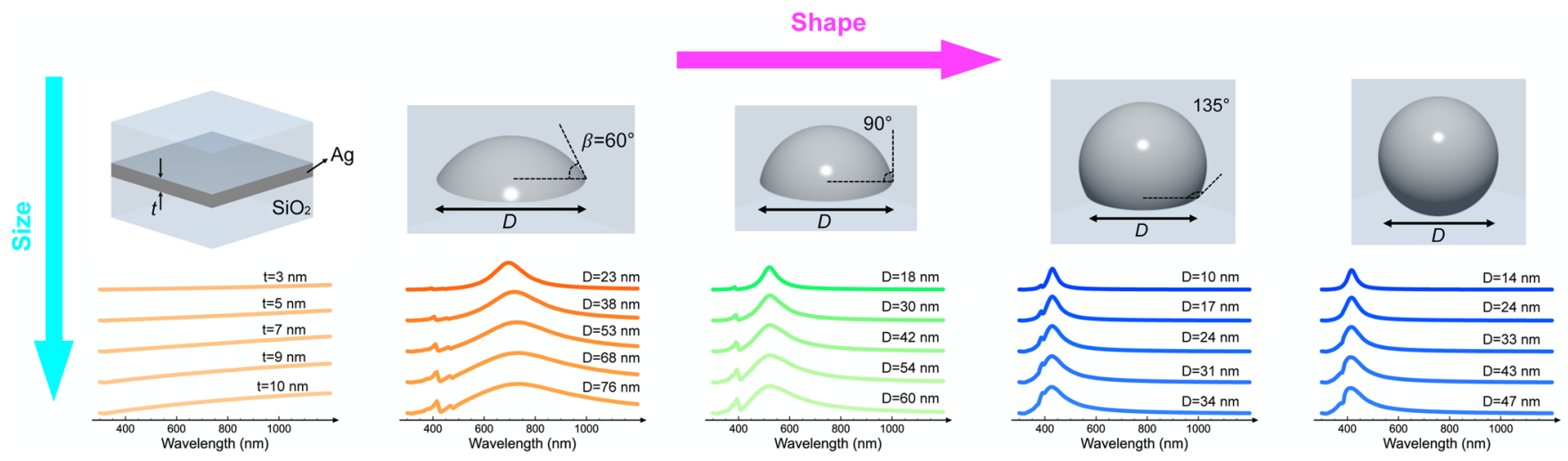

Figure 1. Color tuning through particle size and shape. Top: Geometry and parameters of a silver ultrathin metal film (UTMF) of thickness $t$, along with nanocaps of various shapes and sizes (base diameter $D$ and contact angle $\beta$ ), produced upon dewetting of the film. The metal is embedded in silica. We assume the nanocaps to be distributed in a triangular lattice with spacing $8 t$ and total metal volume equal to that of the homogeneous UTMF in all cases. Bottom: Calculated normal-incidence reflection spectra for particles of varying shape (horizontal direction) and size (vertical). Each curve is given the RGB color extracted from the spectrum that it represents.

(a)

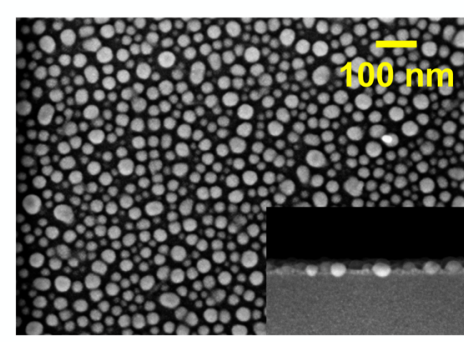

(d)

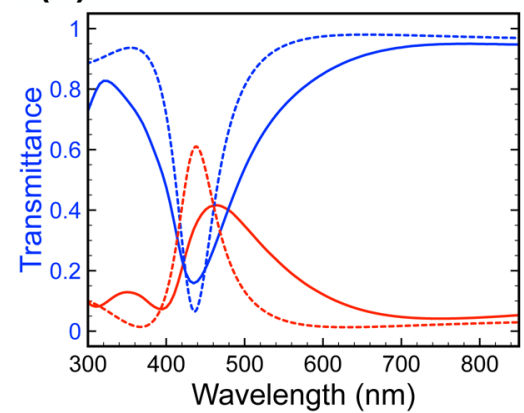

(b)

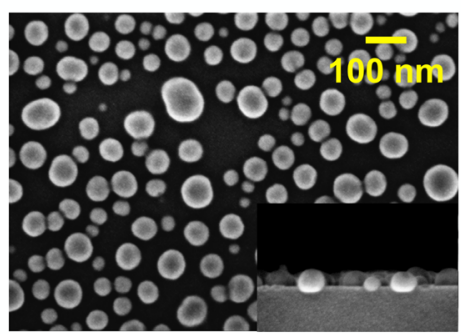

(e)

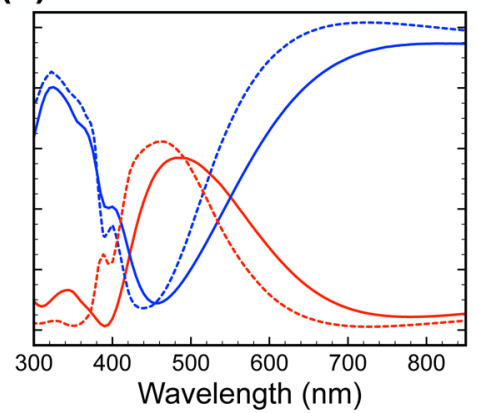

(c)

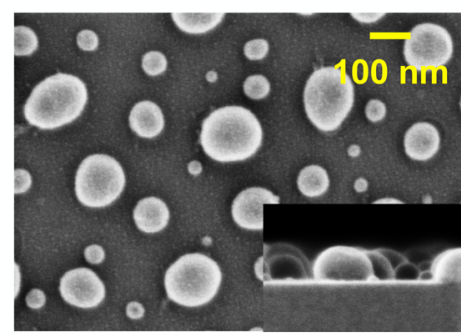

(f)

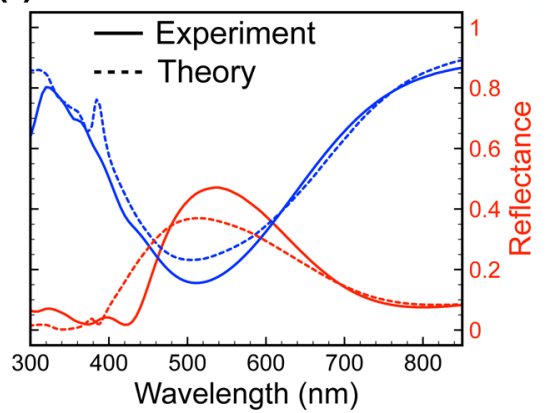

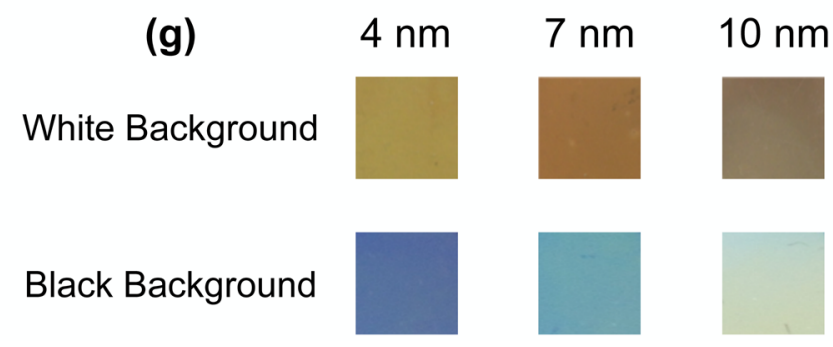

Figure 2. Color tuning through UTMF dewetting. We illustrate this concept with three representative samples produced upon dewetting of silver UTMFs. (a-c) Sample SEM images for different initial silver UTMF thicknesses (i.e., before dewetting; see top labels and also Table 1). (d-f) Measured (solid curves) and calculated (broken curves) normal-incidence transmission (blue curves) and reflection (red curves) spectra for the samples shown in $(\mathrm{a})-(\mathrm{c})$. (g) Photographs for the three samples with measured spectra corresponding to (d) - (f) on white and black backgrounds.

72 approaches, for example, relying on changing particle size. A 73 wide chromatic range is also accessible through varying the 74 initial film thickness. The structures display a high degree of dichroism, with radically different colors on transmission and 75 reflection. We mainly focus on silver-on-silica, for which the 76 contact angle determines the nanocap morphology, although 77 
78 the method can be generally applied to other combinations of 79 metal film and dielectric substrate. We gain further flexibility in 80 color range and spectral absorbance by creating interfering 81 structures with the addition of another properly spaced UTMF.

\section{$82 \square$ RESULTS AND DISCUSSION}

83 The power of changing the contact angle of nanocaps and of a 84 straightforward dewetting process for structural coloring is 85 clearly illustrated by Figure 1, which shows simulated reflection 86 spectra of uniform and dewetted silver UTMFs embedded in 87 silica for typical geometrical parameters similar to those of the 88 actual samples (see below). When the UTMF thickness is 89 increased in the $t=3-10 \mathrm{~nm}$ range, the reflectance gradually 90 grows at long wavelengths. This effect has a minor influence on 91 reflection structural colors, which are mostly faded red (see 92 Figure 1, in which different curves are given the RGB color 93 computed from the spectra that they represent; see Materials 94 and Methods for more details). However, a radical change in 95 color is observed when moving from continuous UTMFs to 96 dewetted nanocaps with different sizes and shapes (contact 97 angle). As we show below, the average base diameter $D$ and 98 contact angle $\beta$ can be controlled by the dewetting temperature, 99 the duration of the thermal treatment used to induce dewetting, 100 and the initial UTMF thickness; so these parameters allow us to 101 generate on-demand bright structural colors, which are in all 102 cases associated with the excitation of LSPRs in the nanocaps. 103 In particular, we observe an excursion along a red-green-blue 104 hue when the contact angle is varied in the $\beta=60-135^{\circ}$ range, 105 driven by a change in plasmon resonance position. Generally, 106 colors become lighter as the nanocap base diameter increases, 107 due to LSPR broadening produced by radiative losses of the 108 individual particles. For illustration, all reflection spectra of 109 Figure 1 are calculated for triangular lattices of spacing $8 t$, with 110 nanocap size determined by the condition that the metal 111 volume is maintained as in the initial film of thickness $t$, which 112 results in the values of the base diameter $D$ indicated by labels. 113 Reflection spectra for spheres are also provided as a reference. 114 Incidentally, we observe similar trends when examining arrays 115 of particles with fixed base diameter but varying contact angle, 116 in which the spacing is adjusted in order to maintain total metal 117 volume as a constant (see Figure S1 in the Supporting 118 Information (SI) for calculations of the transmittance as a 119 function of light wavelength and incidence angle, indicating that 120 LSPR-based coloring is robust against changes of incidence 121 angle). In contrast to previous work, which focused on 122 changing particle size (diameter), our proposed tuning scheme, 123 relying on changing the contact angle, is much wider. 124 Additionally, previous work has also shown that periodic 125 structures can be used to achieve structural coloring, tuning the 126 plasmonic response by changing the period. ${ }^{32-34}$ However, 127 such approaches suffer from either broad resonance 128 features $^{32,33}$ or strong angular dependence of the obtained 129 color. $^{34}$

130 We corroborate the potential of UTMF dewetting for 131 coloring by preparing and optically characterizing samples 132 consisting of silver on silica with different metal thicknesses 133 (see Materials and Methods for fabrication details). A nearly 134 index-matching $150 \mathrm{~nm}$ thick layer of poly(methyl methacry135 late) (PMMA) is deposited on top (2\% difference with the 136 refractive index of the silica substrate; similar results are 137 obtained with silica embedding; see Figure S2 in the SI). The 138 results are summarized in Figure 2. In particular, Figure $2 a-c$ 139 shows SEM images of three samples, which produce the transmission and reflection spectra plotted in Figure $2 \mathrm{~d}-\mathrm{f}$, and 140 these in turn generate the colors shown in Figure 2g.

As a rule, a larger initial metal thickness gives rise to lower 142 particle density and larger particle size (Figure 2a-c). This 143 increase in size switches on retardation, which in turn produces 144 plasmon broadenings and red-shifts (Figure 2d-f). Similar 145 conclusions can be extracted from samples of nanocaps exposed 146 to air (see Figure S2 in the SI), but now the LSPRs are blue- 147 shifted with respect to those covered with PMMA. The 148 dielectric protection also prevents silver oxidation: we have 149 verified their stable optical properties over a period of several 150 months.

Figure $2 \mathrm{~g}$ displays photographs taken from these three 152 samples (Figure 2d-f). A large contrast in structural colors 153 depending on whether the samples are on white or black 154 background is evident.

We seek further understanding of our measurements (Figure 156 $2 \mathrm{~d}-\mathrm{f}$, solid curves) by formulating an analytical theoretical 157 model (broken curves), which is in excellent agreement with 158 experiment in the details of the reflection/transmission spectra 159 (Figure $2 \mathrm{~d}-\mathrm{f}$ ). The model uses as input the geometrical 160 parameters retrieved upon inspection of the sample SEM 161 images (Figure $2 \mathrm{a}-\mathrm{c}$ ), which are summarized in Table 1 . We $162 \mathrm{tl}$

Table 1. Statistical Parameters for Three Fabricated Samples with Different Initial Silver UTMF Thicknesses, as Extracted by SEM Image Analysis ${ }^{a}$

$\begin{array}{cccccc}\begin{array}{c}\text { initial film } \\ \text { thickness } \\ (\mathrm{nm})\end{array} & \begin{array}{c}\text { particle } \\ \text { density } \\ \left(\mu \mathrm{m}^{-2}\right)\end{array} & \begin{array}{c}\text { average } \\ \text { base } \\ \text { diameter } \\ (\mathrm{nm})\end{array} & \begin{array}{c}\text { diameter } \\ \text { std }(\mathrm{nm})\end{array} & \begin{array}{c}\text { average } \\ \text { height } \\ (\mathrm{nm})\end{array} & \begin{array}{c}\text { estimated } \\ \text { contact } \\ \text { angle }(\mathrm{deg})\end{array} \\ 4 & 805 & 20 & 9 & 16 & 116 \\ 7 & 210 & 34 & 14 & 35 & 128 \\ 10 & 35 & 82 & 30 & 71 & 120\end{array}$

${ }^{a}$ See Figure $2 \mathrm{a}-\mathrm{c}$. The contact angle is estimated from the height and base diameter assuming spherical nanocap profiles.

describe the particles as spherical nanocaps similar to those of 163 Figure 1 and assume a fixed value of the silver-on-silica contact 164 angle $\beta=120^{\circ}$. The optical response of the nanocaps is 165 reduced to their average anisotropic polarizability (for axial and 166 transversal directions), which we combine with the dynamical 167 interaction between them, taking into account their random 168 distribution, to produce reflection and transmission coefficients 169 (see Materials and Methods for more details). This model 170 produces satisfactory results compared with experiment (Figure 171 $2 \mathrm{~d}-\mathrm{f}$ ), except for the discrepancy in plasmon width of the 172 thinner sample (Figure 2d), where the particle details are at the 173 limit of the SEM spatial resolution, which probably affects the 174 determination of statistic geometrical parameters. 175

The optical response of our samples emerges from the 176 properties of the individual nanocaps and their LSPRs, which 177 we investigate in more detail in Figure 3. In particular, the $178 \mathrm{f} 3$ dependence on contact angle $\beta$ is summarized in Figure $3 a-c 179$ for small nanocaps (base diameter $D=10 \mathrm{~nm}$ ), for which 180 retardation can be neglected. The response in the spectral range 181 under consideration is dominated by the lowest order axial and 182 transversal dipolar plasmons, whose spectral position and 183 weight are fully captured by two parameters in the electrostatic 184 limit, which we extract from boundary-element method 185 electromagnetic simulations ${ }^{25}$ (BEM; see methods): the value 186 of the metal permittivity at which the plasmon shows up 187 (Figure 3a) and the dipolar transition strength (Figure 3b). The 188 
(a)

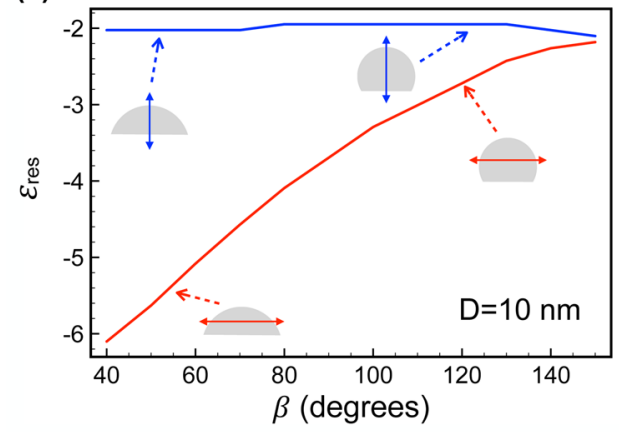

(b)

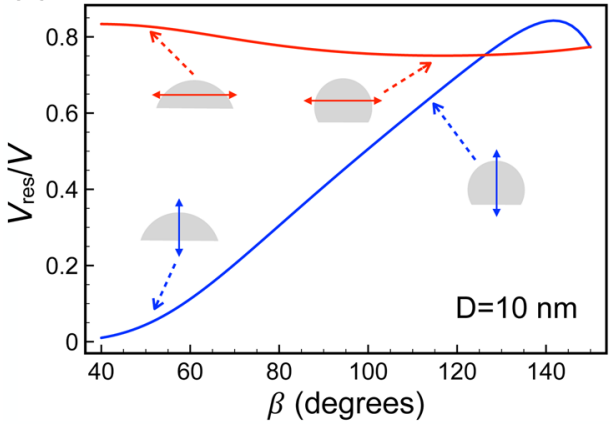

(c)

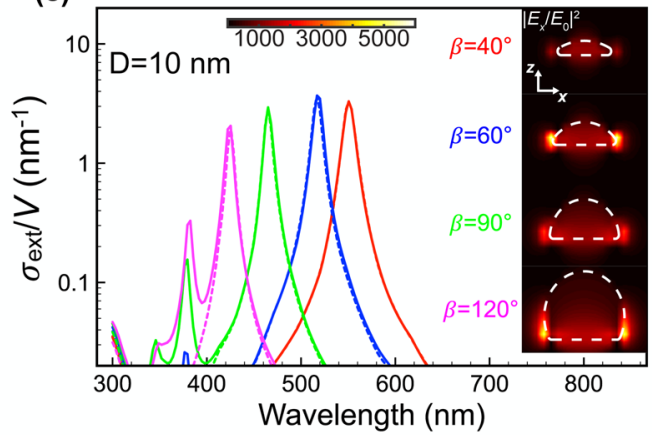

(d)

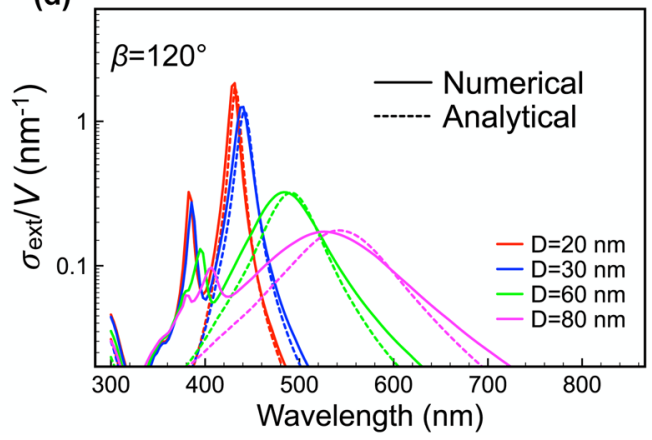

Figure 3. Plasmons in spherical nanocaps. (a) Resonant value of the permittivity for which a dipolar plasmon is sustained by nanocaps of varying shape (i.e., as a function of contact angle $\beta$ ). Both transversal and axial polarizations are considered (see insets and double arrows). (b) Fractional contribution to the spectral weight for the plasmons considered in (a). (c) Normalized extinction spectra of these nanocaps for light incident along the axial direction. Numerical electromagnetic simulations (solid curves) are well reproduced by a simple analytical model (dashed curves; see Materials and Methods) based on the dominant transversal plasmon. (d) Same as (c) for nanocaps of fixed shape $\left(\beta=120^{\circ}\right)$ and different larger sizes (the legend indicates the base diameter legend $D$ ), illustrating the ability of our model to cope with retardation effects (see Materials and Methods).

189 latter is expressed in terms of a fractional volume associated 190 with the resonance, and it amounts to the fraction that it 191 contributes to the integral of the extinction cross-section over 192 frequency. These two parameters are all the input that we need 193 to analytically describe the polarizability of the particles (see 194 Materials and Methods), from which we calculate the cross195 section represented in Figure 3c (broken curves). Despite the 196 simplicity of this method, we find a remarkable agreement of 197 the spectra with BEM simulations (broken curves). Notice that 198 under light incidence along the particle axis the transversal 199 plasmon dominates the response. Near-field plots associated 200 with this mode reveal light confinement and enhancement near 201 the edge of the particle base (see insets to Figure 3c). Unlike 202 the axial plasmon, the transversal one has a strong dependence 203 on contact angle (Figure 3a), whereas its spectral weight 204 remains at a high level of $\sim 0.8$ (Figure $3 \mathrm{~b}$ ). This explains the 205 weak dependence of the optical properties of our samples on 206 incidence angle. Nevertheless, the axial plasmon can also be 207 excited in our nanostructures at larger incidence angle, 208 emerging as a feature at shorter wavelength (see Figure S1 in 209 the SI).

210 When the particle size increases, retardation effects become 211 important, leading to plasmon red-shifting and broadening. 212 Both of these effects can be easily accounted for in a modified 213 analytical polarizability that we present in Materials and 214 Methods. The results are in excellent agreement with 215 electromagnetic BEM simulations, as illustrated in Figure 3d 216 for nanocaps of shape and sizes similar to those involved in the 217 measured samples of Figure 2. Additionally, the dominant 218 plasmon feature in the spectra is expected to blue-shift with increasing contact angle (see Figure 3c), as the particle aspect 219 ratio is reduced. This result is fully corroborated by 220 measurements on additional samples in which a treatment of 221 the surface can produce a change in contact angle (see Figure 222 S3 in the SI). Further comparisons between numerical and 223 theoretical results for nanocaps with other contact angles are 224 offered in Figure S4 of the SI.

Optical interference can also produce structural colors, 226 adding an extra knob to further tune the chromatic suite 227 achieved through metal dewetting. We test this principle by 228 adding an extra nickel layer of thickness varied from $d=3 \mathrm{~nm} 229$ (transparent) to $40 \mathrm{~nm}$ (opaque). In particular, we show in 230 Figure $4 \mathrm{a}$ results for samples produced by this procedure $231 \mathrm{f} 4$ starting with a dewetted $7 \mathrm{~nm}$ silver film, covered with $150 \mathrm{~nm} 232$ of PMMA. A clear asymmetric plasmonic coloring effect is 233 observed in measurements from the two sides of the sample 234 (front and back, see scheme in Figure 4a). Transmittance from 235 both sides is identical in virtue of reciprocity, as corroborated in 236 both measurements and simulations (see Figure $4 \mathrm{~b}$ and also 237 Figure S5 in the SI), and it eventually evolves toward negligible 238 values as the nickel eventually becomes optically thick. In 239 contrast, there are clear differences in absorbance from both 240 sides: front-side absorption increases with nickel thickness $d$ in 241 the $300-550 \mathrm{~nm}$ wavelength range, reaching values above $85 \%, 242$ essentially as a result of a Salisbury screen effect; ${ }^{35}$ however, 243 back-side absorption decreases with increasing $d$, attenuating 244 the effect of the dewetted silver layer and eventually saturating 245 at the bulk nickel surface absorption level. The chromatic 246 variation produced upon nickel deposition is illustrated by 247 photographs in Figure 4c. We note that both in the spectra and 248 
(a)

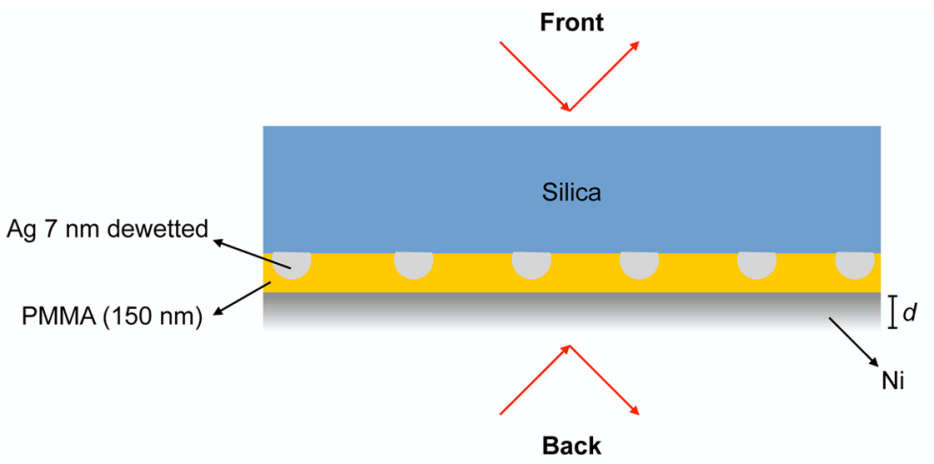

(c)

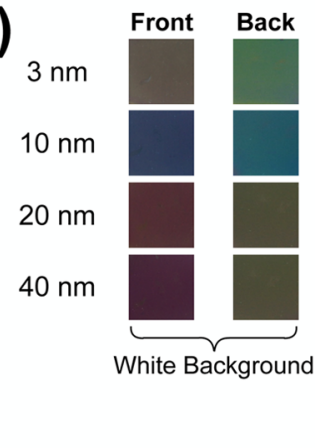

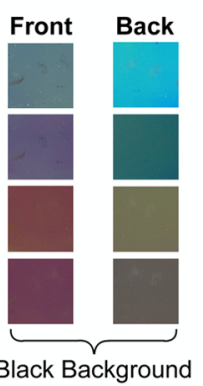
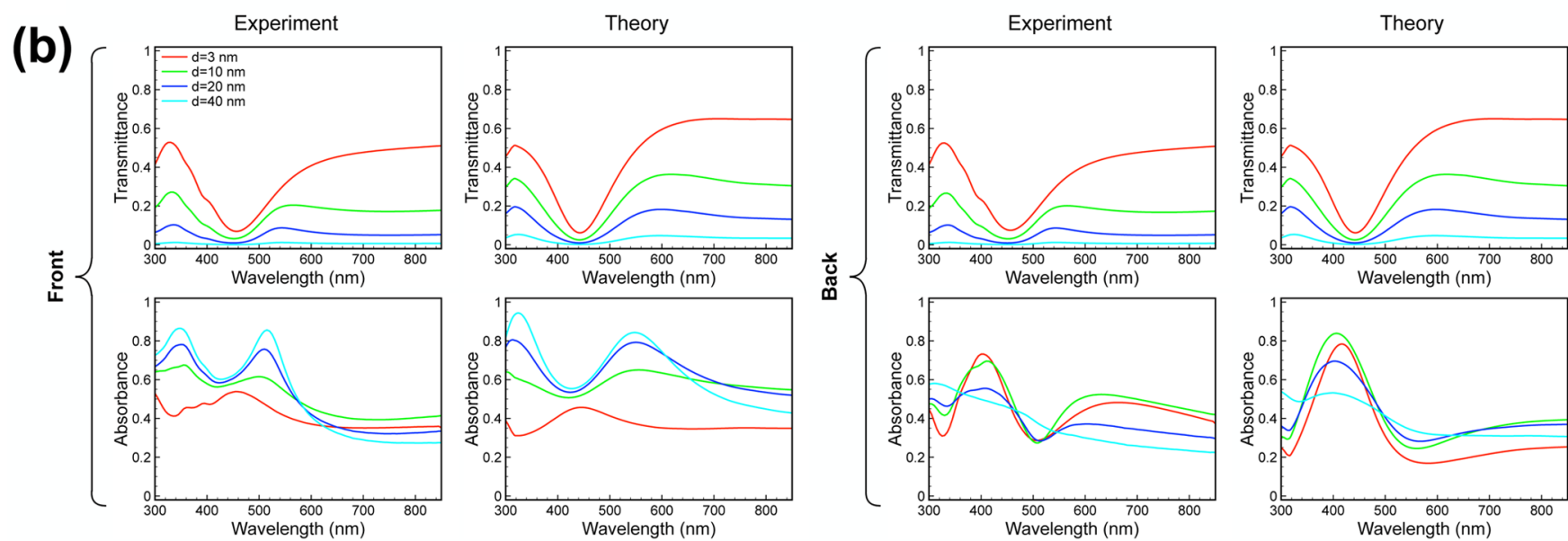

Figure 4. Additional color tuning through interference. (a) A nickel UTMF is deposited on a $150 \mathrm{~nm}$ PMMA spacer embedding the silver nanocaps that are previously obtained upon dewetting a $7 \mathrm{~nm}$ silver film. (b) Measured and calculated transmission and absorption spectra for normalincidence illumination from the back and front sides of the structure (see (a)). We consider different nickel thicknesses in the $d=3-40 \mathrm{~nm}$ range. (c) Photographs of the samples whose measured absorption (reflection) spectra are shown in (b), taken on white and black backgrounds.
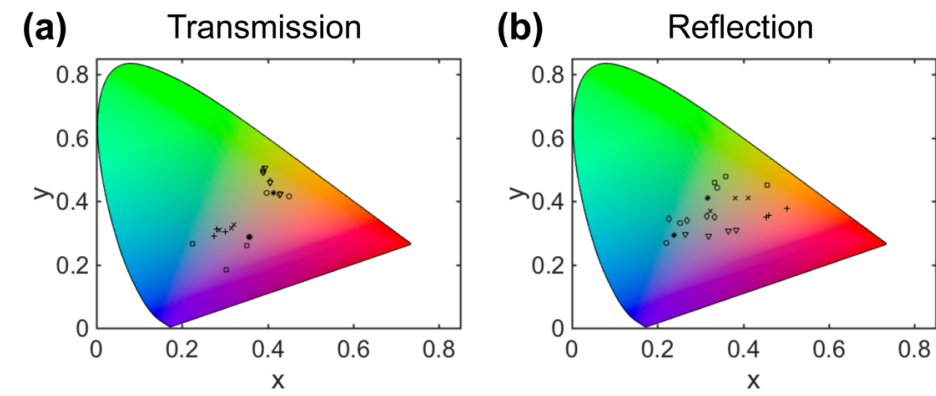

- Ag on silica (Fig. 2)

+ Au on silica (Fig. S6)

* Cu on silica (Fig. S6)

- Ag on sapphire (Fig. S7)

- Ag on coated silica (Fig. S3)

- Fig. 4 (Front)

- Fig. 4 (Back)

Figure 5. Position of the colors in our samples on the CIE 1931 map. We show the colors associated with our experimental spectra (see labels), as obtained for a D65 illumination light source.

249 in the resulting colors our simple theory compares reasonably 250 well with experiment, including in this case the effect of 251 multiple reflections at the different interfaces of the sample.

\section{$252 \square$ CONCLUSION}

253 In summary, we have demonstrated that arrays of metal 254 nanocaps created via a low-cost, lithography-free dewetting 255 process display intense colors as a result of the excitation of 256 localized surface plasmons in the particles. A wide chromatic 257 range is accessible through careful control of the initial metal 258 thickness and the dewetting conditions (temperature and 259 duration), which ultimately defines the size, density, and 260 contact angle of the resulting nanoparticles. Colors can be 261 actually customized over a sizable range of the visible spectrum for a wide range of incidence angles, as shown in Figure 5, $262 \mathrm{fs}$ where we represent the colors associated with our obtained 263 experimental spectra superimposed on the Commission 264 Internationale de l'Elcairage (CIE) 1931 color map. For 265 example, we observe structures that go from reflective 266 (scattered) red to green and blue, suggesting the potential for 267 large-scale manufacturing of structurally colored glass windows, 268 optical filters, and display panels. ${ }^{36}$ The addition of an extra 269 ultrathin metal film produces interference with the dewetted 270 film, leading to further control over the chromatic properties. 271 The combination of continuous and dewetted metal layers thus 272 holds great potential to achieve full control over the visible 273 spectral properties of thin films, which are accessible though 274 industrially scalable, economic fabrication processes. Our 275 
276 results can be readily extrapolated to other spectral regimes 277 through a choice of appropriate materials (e.g., aluminum for 278 the UV).

\section{MATERIALS AND METHODS}

280 Ultrathin Metal Film Deposition and Dewetting. Silver 281 ultrathin films are deposited on the silica substrate using a 282 magnetron sputtering system (ATC Orion 8, AJA International, 283 Inc. or KDF $903 \mathrm{i}$ ). The coated substrates are then subjected to 284 rapid thermal annealing in a Tsunami RTP-600S at a 285 temperature of $750{ }^{\circ} \mathrm{C}$ for about $90 \mathrm{~s}$ under a nitrogen flow 286 of $1 \mathrm{~atm}$. In this way the ultrathin silver films are dewetted into 287 nanocaps. The PMMA overlayer is deposited through spin288 coating.

289 Sample Characterization. Optical transmittance and 290 reflectance are measured using a UV-vis-NIR spectropho291 tometer (PerkinElmer Lambda 950). The morphology of the 292 nanocaps is examined with a field-emission scanning electron 293 microscope (FEG-SEM, Inspect F, FEI Systems).

294 Simulation of Individual Nanocaps. The optical 295 response of individual nanocaps is simulated using BEM. ${ }^{25}$ 296 The metal dielectric function is taken from tabulated data, ${ }^{26}$ 297 whereas a homogeneous surrounding medium is assumed with 298 constant permittivity $\epsilon_{\mathrm{s}}=2.13$ similar to that of silica and 299 PMMA in the spectral region under investigation. The particle 300 geometry is determined by its base diameter $D$ and contact 301 angle with the substrate $\beta$, while the edges are smoothed with a 302 rounded radius of $0.5 \mathrm{~nm}$. These simulations yield the particle 303 extinction cross-section and its scattering matrix used to 304 simulate arrays.

305 Average Polarizability of Nanocaps in the Actual 306 Samples. For a given sample, the contact angle is roughly 307 constant, and in particular, it takes the value $\beta=120^{\circ}$ for silver 308 on silica. However, the particle size has a finite size distribution, 309 which we describe through a Gaussian,

$$
P(D)=\frac{1}{\sqrt{2 \pi} \sigma} \mathrm{e}^{-(D-\bar{D})^{2} / 2 \sigma^{2}}
$$

310 as a function of the base diameter $D$, centered around its 311 average $\bar{D}$ with standard deviation $\sigma$. These parameters $(\bar{D}$ and $312 \sigma$ ) depend on the initial UTMF thickness, as shown in Table 1. 313 We then describe the particles through their average polar314 izability:

$$
\bar{\alpha}_{\|, \perp}(\omega)=-\frac{1}{k^{2}} \int f_{\|, \perp}(\omega, D) P(D) \mathrm{dD}
$$

315 where $f_{\|, \perp}(\omega, D)$ is the far-field amplitude of individual 316 nanocaps for transversal $(\perp)$ and axial $(\|)$ incident field 317 polarization, calculated with BEM as noted above.

318 Analytical Model for Individual Nanocaps. We 319 elaborate an analytical model based upon the dipolar response 320 of the particles, supplemented to incorporate retardation 321 effects, which are important for large sizes, although the 322 particle diameters under consideration are still small compared 323 with the wavelength, so that the dipolar response becomes 324 dominant. In the electrostatic limit, the particle polarizability 325 can be written as ${ }^{27}$

$$
\alpha_{\mathrm{es}}(\omega)=\frac{1}{4 \pi \epsilon_{\mathrm{s}}} \sum_{j} V_{j}\left[\frac{1}{\epsilon(\omega)-\epsilon_{\mathrm{s}}}-\frac{1}{\epsilon_{\mathrm{s}}\left(\epsilon_{j}-1\right)}\right]^{-1}
$$

where $\epsilon(\omega)$ and $\epsilon_{\mathrm{s}}$ are the permittivities of the metal in the 326 particle and the dielectric host, respectively. Here, the sum 327 extends over the electrostatic eigenmodes of the system, which 328 are identified by eigenvalues $\epsilon_{j}$ and contribute to the 329 polarizability with a partial volume $V_{j}$ (the sum of $V_{j}^{\prime}$ s is 330 equal to the particle volume). Retardation produces two effects: 331 (1) radiative damping, which we incorporate through the well- 332 known prescription ${ }^{28}$

$$
\alpha(\omega)=\frac{1}{\alpha_{\mathrm{es}}{ }^{-1}-2 \mathrm{i} k^{3} / 3}
$$

where $k=\omega / c$; and (2) plasmon red-shifts, which we 334 phenomenologically describe through the substitution

$$
\epsilon_{j} \rightarrow \frac{\epsilon_{j}}{\cos (k \xi D)}+\left[1-\frac{1}{\cos (k \xi D)}\right] \frac{1}{\epsilon_{s}}
$$

where $\xi$ is a scaling factor. Finally, we compare the extinction 336 cross-section computed with BEM (see above) with the 337 analytical cross-section $4 \pi k \operatorname{Im}\{\alpha(\omega)\}$ in order to extract fitting 338 parameters $\epsilon_{j}, V_{j}$, and $\xi$, which depend only on the geometrical 339 contact angle $\beta$, but not on material composition and 340 frequency. In particular, we find $\xi=0.75$ for the contact 341 angle $\beta=120^{\circ}$ of silver on silica. In practice, we retain only the 342 lowest order dipolar mode for polarization directions either 343 parallel to the nanocap base (transverse) or along its rotation 344 axis (axial).

345

Simulation of Periodic Nanocap Arrays. We use the 346 layer-KKR method ${ }^{29}$ to simulate the response of periodic 347 particle arrays (see Figure S1 in the SI). A rigorous solution of 348 Maxwell's equations is then obtained by describing the particles 349 through their scattering matrix (i.e., their multipolar response), 350 as obtained with BEM (see above).

Analytical Model for Disordered Nanocap Arrays. We 352 formulate an analytical model of the response of the samples by 353 describing the nanocaps through the average polarizability $\bar{\alpha} 354$ (see above), following similar methods to those reported 355 elsewhere. ${ }^{30,31}$ In particular, the reflection and transmission 356 coefficients for p-polarized light with angle of incidence $\theta 357$ reduce to

$$
\begin{aligned}
& r=\frac{\mathrm{i} 2 \pi k \rho}{\cos \theta}\left(\frac{-\cos ^{2} \theta}{\bar{\alpha}_{\|}^{-1}-G_{\|}}+\frac{\sin ^{2} \theta}{\bar{\alpha}_{\perp}^{-1}-G_{\perp}}\right) \\
& t=1-r+\frac{\mathrm{i} 2 \pi k \rho}{\cos \theta} \frac{2 \sin ^{2} \theta}{\bar{\alpha}_{\perp}^{-1}-G_{\perp}}
\end{aligned}
$$

where $\rho$ is the particle density, whereas $G_{\|, \perp}$ are the dipole- 359 dipole interaction lattice sums. The latter allows the expression 360

$$
G_{\|, \perp}=\int \frac{d^{2} \mathbf{Q}}{(2 \pi)^{2}}\left[\frac{1}{\rho} S\left(\mathbf{Q}-\mathbf{k}_{\|}\right)-1\right] \mathcal{G}_{\|, \perp}(\mathbf{Q})
$$

in terms of the momentum representation of the dipole-dipole 361 in ter a c tio n c o m p o n e n t s 362 $\mathcal{G}_{\perp}(\mathbf{Q})=2 \pi \mathrm{i} k_{z} Q_{x}{ }^{2} / Q^{2}+2 \pi \mathrm{i}\left(k^{2} / k_{z}\right)\left(1-Q_{x}{ }^{2} / Q^{2}\right) \quad$ a n d $\mathcal{G}_{\|}(\mathbf{Q})=2 \pi \mathrm{i} Q^{2} / k_{z}$, where $k_{z}=\sqrt{k^{2}-Q^{2}}$, as extracted for both interacting dipoles oriented either perpendicular or 363 parallel to the surface normal, respectively. The lattice sums 364 also depend on the surface projection of the incident light wave 365 vector $\mathbf{k}_{\|}$and the structure factor $S(\mathbf{Q})=\sum_{\mathbf{R}} \exp (\mathrm{iQ} \cdot \mathbf{R})$, where 366 the sum runs over particle positions. More precisely, we have 367 
$368 S(\mathbf{Q})=(2 \pi \rho)^{2} \delta(\mathbf{Q})+\rho$ for a completely disordered array and $369 S(\mathbf{Q})=(2 \pi \rho)^{2} \sum_{\mathbf{g}} \delta(\mathbf{Q}-\mathbf{g})$ for a periodic one, where $\mathbf{g}$ runs 370 over reciprocal lattice vectors. Theory curves in Figures 2 and 4 371 are obtained assuming random particle distributions.

372 RGB Color. We show RGB colors in several figures, as 373 extracted from measured and calculated spectra, assuming a 374 flat-spectrum illumination. RGB parameters are obtained 375 simply by projection on the spectra of the three pigments 376 that define this color scheme.

\section{ASSOCIATED CONTENT}

\section{S Supporting Information}

379 The Supporting Information is available free of charge on the 380 ACS Publications website at DOI: 10.1021/acsphoto381 nics.6b00090.

382 Further simulations and experiments and, in particular, 383 calculations of the transmittance of ordered and 384 disordered planar particle arrays for fixed particle size 385 and different contact angles (Figure S1); measured 386 reflection and transmission spectra for silver nanocaps 387 with different surrounding media (Figure S2) and 388 contact angles (Figure S3 and Table S1); an extension 389 of Figure 3d to cover more contact angles (Figure S4); 390 the reflectance spectra corresponding to the samples of 391 Figure 4b (Figure S5); and measured spectra for gold 392 and copper on silica (Figure S6) and silver on sapphire 393 (Figure S7) (PDF)

\section{AUTHOR INFORMATION}

\section{Corresponding Authors}

396 *E-mail: javier.garciadeabajo@icfo.es.

397 *E-mail: valerio.pruneri@icfo.es.

\section{Notes}

399 The authors declare no competing financial interest.

\section{ACKNOWLEDGMENTS}

401 This work has been supported in part by Spanish MINECO 402 (MAT2014-59096-P and SEV2015-0522), Fundació Privada 403 Cellex, the European Commission (Graphene Flagship 404 CNECT-ICT-604391 and FP7-ICT-2013-613024-GRASP), 405 AGAUR (2014 SGR 1400 and 1623), and the Fondo Europeo 406 de Desarrollo Regional (FEDER, TEC2013-46168-R).

\section{$407 \square$ REFERENCES}

408 (1) Freestone, I.; Meeks, N.; Sax, M.; Higgitt, C. The Lycurgus Cup 409 A Roman nanotechnology. Gold Bulletin 2007, 40, 270-277.

410 (2) Kinoshita, S.; Yoshioka, S.; Miyazaki, J. Physics of structural 411 colors. Rep. Prog. Phys. 2008, 71, 076401.

412 (3) Inoue, D.; Miura, A.; Nomura, T.; Fujikawa, H.; Sato, K.; Ikeda, 413 N.; Tsuya, D.; Sugimoto, Y.; Koide, Y. Polarization independent visible 414 color filter comprising an aluminum film with surface-plasmon 415 enhanced transmission through a subwavelength array of holes. Appl. 416 Phys. Lett. 2011, 98, 093113.

417 (4) Lee, H.-S.; Yoon, Y.-T.; Lee, S.-s.; Kim, S.-H.; Lee, K.-D. Color 418 filter based on a subwavelength patterned metal grating. Opt. Express 419 2007, 15, 15457-15463.

420 (5) Roberts, A. S.; Pors, A.; Albrektsen, O.; Bozhevolnyi, S. I. 421 Subwavelength plasmonic color printing protected for ambient use. 422 Nano Lett. 2014, 14, 783-787.

423 (6) Si, G.; Zhao, Y.; Lv, J.; Lu, M.; Wang, F.; Liu, H.; Xiang, N.; 424 Huang, T. J.; Danner, A. J.; Teng, J.; Liu, Y. J. Reflective plasmonic 425 color filters based on lithographically patterned silver nanorod arrays. 426 Nanoscale 2013, 5, 6243-6248.
(7) Xu, T.; Wu, Y.-K.; Luo, X.; Guo, L. J. Plasmonic nanoresonators 427 for high-resolution colour filtering and spectral imaging. Nat. Commun. 428 2010, 1, 59.

(8) Kumar, K.; Duan, H.; Hegde, R. S.; Koh, S. C. W.; Wei, J. N.; 430 Yang, J. K. W. Printing colour at the optical diffraction limit. Nat. 431 Nanotechnol. 2012, 7, 557-561.

(9) Cheng, F.; Gao, J.; Luk, T. S.; Yang, X. Structural color printing 433 based on plasmonic metasurfaces of perfect light absorption. Sci. Rep. 434 2015, 5, 11045 .

(10) Li, Z.; Clark, A. W.; Cooper, J. M. Dual Color Plasmonic Pixels 436 Create a Polarization Controlled Nano Color Palette. ACS Nano 2016, 437 10, 492-498.

(11) Clark, A. W.; Cooper, J. M. Plasmon Shaping by using Protein 439 Nanoarrays and Molecular Lithography to Engineer Structural Color. 440 Angew. Chem. 2012, 124, 3622-3626.

(12) Olson, J.; Manjavacas, A.; Liu, L.; Chang, W.-S.; Foerster, B.; 442 King, N. S.; Knight, M. W.; Nordlander, P.; Halas, N. J.; Link, S. Vivid, 443 full-color aluminum plasmonic pixels. Proc. Natl. Acad. Sci. U. S. A. 444 2014, 111, 14348-14353.

(13) Clausen, J. S.; Højlund-Nielsen, E.; Christiansen, A. B.; Yazdi, 446 S.; Grajower, M.; Taha, H.; Levy, U.; Kristensen, A.; Mortensen, N. A. 447 Plasmonic metasurfaces for coloration of plastic consumer products. 448 Nano Lett. 2014, 14, 4499-4504.

(14) Macleod, H. A. Thin-Film Optical Filters; CRC Press, 2001. 450

(15) Lichtman, J. W.; Conchello, J. A. Fluorescence microscopy. Nat. 451 Methods 2005, 2, 910-919.

(16) Sampsell, J. B.; Florence, J. M. Projection display system for 453 reflective light valves. U.S. Patent US6,113,239, 2000.

(17) Li, Z.; Butun, S.; Aydin, K. Large-Area, Lithography-Free Super 455 Absorbers and Color Filters at Visible Frequencies Using Ultrathin 456 Metallic Films. ACS Photonics 2015, 2, 183-188.

457

(18) Kats, M. A.; Blanchard, R.; Genevet, P.; Capasso, F. Nanometre 458 optical coatings based on strong interference effects in highly 459 absorbing media. Nat. Mater. 2013, 12, 20-24. 460

(19) Nassau, K. The Physics and Chemistry of Color: The Fifteen Causes 461 of Color; Wiley-VCH: New York, 2001.

(20) Yan, M.; Dai, J.; Qiu, M. Lithography-free broadband visible 463 light absorber based on a mono-layer of gold nanoparticles. J. Opt. 464 2014, 16, 025002.

(21) Mazumder, P.; Jiang, Y.; Baker, D.; Carrilero, A.; Tulli, D.; 466 Infante, D.; Hunt, A. T.; Pruneri, V. Superomniphobic, transparent, 467 and antireflection surfaces based on hierarchical nanostructures. Nano 468 Lett. 2014, 14, 4677-4681.

(22) Tulli, D.; Hart, S. D.; Mazumder, P.; Carrilero, A.; Tian, L.; 470 Koch, K. W.; Yongsunthon, R.; Piech, G. A.; Pruneri, V. Monolithically 471 Integrated Micro- and Nanostructured Glass Surface with Antiglare, 472 Antireflection, and Superhydrophobic Properties. ACS Appl. Mater. 473 Interfaces 2014, 14, 11198-11203. 474

(23) Thompson, C. V. Solid-state dewetting of thin films. Annu. Rev. 475 Mater. Res. 2012, 42, 399-434. 476

(24) Infante, D.; Koch, K. W.; Mazumder, P.; Tian, L.; Carrilero, A.; 477 Tulli, D.; Baker, D.; Pruneri, V. Durable, superhydrophobic, 478 antireflection, and low haze glass surfaces using scalable metal 479 dewetting nanostructuring. Nano Res. 2013, 6, 429-440.

(25) García de Abajo, F. J.; Howie, A. Retarded field calculation of 481 electron energy loss in inhomogeneous dielectrics. Phys. Rev. B: 482 Condens. Matter Mater. Phys. 2002, 65, 115418.

(26) Johnson, P. B.; Christy, R. W. Optical constants of the noble 484 metals. Phys. Rev. B 1972, 6, 4370-4379.

(27) Solís, D. M.; Taboada, J. M.; Obelleiro, F.; Liz-Marzán, L. M.; 486 García de Abajo, F. J. Toward ultimate nanoplasmonics modeling. ACS 487 Nano 2014, 8, 7559-7570.

(28) Draine, B. T. The discrete-dipole approximation and its 489 application to interstellar graphite grains. Astrophys. J. 1988, 333, 848- 490 872.

(29) Stefanou, N.; Yannopapas, V.; Modinos, A. MULTEM 2: A new 492 version of the program for transmission and band-structure 493 calculations of photonic crystals. Comput. Phys. Commun. 2000, 132, 494 189-196. 
496 (30) García de Abajo, F. J. Colloquium: Light scattering by particle 497 and hole arrays. Rev. Mod. Phys. 2007, 79, 1267-1290.

498 (31) Stauber, G. G.; García de Abajo, F. J. Extraordinary absorption 499 of decorated undoped graphene. Phys. Rev. Lett. 2014, 112, 077401.

500 (32) Shrestha, V. R.; Lee, S.-S.; Kim, E.-S.; Choi, D.-Y. Aluminum 501 plasmonics based highly transmissive polarization-independent sub502 tractive color filters exploiting a nanopatch array. Nano Lett. 2014, 14, $5036672-6678$.

504 (33) Tan, S. J.; Zhang, L.; Zhu, D.; Goh, X. M.; Wang, Y. M.; Kumar, 505 K.; Qiu, C.-W.; Yang, J. K. Plasmonic color palettes for photorealistic 506 printing with aluminum nanostructures. Nano Lett. 2014, 14, 40235074029.

508 (34) Shen, Y.; Rinnerbauer, V.; Wang, I.; Stelmakh, V.; 509 Joannopoulos, J. D.; Soljacic, M. Structural colors from Fano 510 resonances. ACS Photonics 2015, 2, 27-32.

511 (35) Munk, B. A. Frequency Selective Surfaces: Theory and Design; John 512 Wiley \& Sons, 2005.

513 (36) Hsu, C. W.; Zhen, B.; Qiu, W.; Shapira, O.; DeLacy, B. G.; 514 Joannopoulos, J. D.; Soljačić, M. Transparent displays enabled by 515 resonant nanoparticle scattering. Nat. Commun. 2014, 5, 3152. 